\title{
Fuzzy Logic in the APOS/ACE Instructional Treatment for Mathematics
}

\author{
Michael Gr. Voskoglou* \\ School of Technological Applications, Graduate Technological Educational Institute (T. E. I.) of Western Greece, Patras, Greece \\ *Corresponding author: mvosk@hol.gr
}

Received February 08, 2015; Revised March 01, 2015; Accepted March 04, 2015

\begin{abstract}
In this paper principles of fuzzy logic are introduced for comparing the performance of two student groups concerning the comprehension of real numbers in general and of irrational numbers in particular. The first group was taught the subject in the traditional way (control group), while the APOS/ACE instructional treatment was applied for the second group (experimental group). The two groups are represented as fuzzy subsets of the set of the grades (from A to F) achieved by the students in a pre-instructional and a post-instructional test and the centroid defuzzification technique is applied on comparing their performances. The results of our classroom experiments show that the application of the APOS/ACE approach can effectively help students to enlist the real numbers in a powerful cognitive schema including all the basic sets of numbers.
\end{abstract}

Keywords: Fuzzy sets, centroid defuzzification technique, teaching and learning the real numbers, APOS/ACE theory

Cite This Article: Michael Gr. Voskoglou, "Fuzzy Logic in the APOS/ACE Instructional Treatment for Mathematics.” American Journal of Educational Research, vol. 3, no. 3 (2015): 330-339. doi: 10.12691/education3-3-12.

\section{Introduction}

There used to be a tradition in science and engineering of turning to probability theory when one is faced with a problem in which uncertainty plays a significant role. This transition was justified when there were no alternative tools for dealing with the uncertainty. Today this is no longer the case. Fuzzy logic, which is based on fuzzy sets theory introduced by Zadeh [22] in 1965, provides a rich and meaningful addition to standard logic. The applications which may be generated from or adapted to fuzzy logic are wide-ranging and provide the opportunity for modelling under conditions which are inherently imprecisely defined, despite the concerns of classical logicians. Many systems may be modelled, simulated and even replicated with the help of fuzzy logic, not the least of which are human cognitive systems (e.g. [3,6,8,9,11-17], etc)

A real test of the effectiveness of an approach to uncertainty is the capability to solve problems which involve different facets of uncertainty. Fuzzy logic offers a much higher problem solving capability than standard probability theory. Most importantly, it opens the door to construction of mathematical solutions of computational problems which are stated in a natural language. In contrast, standard probability theory does not have this capability, a fact which is one of its principal limitations.

The methods of assessing the individuals' performance usually applied in practice are based on principles of the bivalent logic (yes-no). However these methods are not probably the most suitable ones in ambiguous cases characterized by a degree of uncertainty. On the contrary, fuzzy logic, due to its nature of including multiple values, offers a wider and richer field of resources for this purpose. This gave us several times in the past the impulsion to introduce principles of fuzzy logic for assessing the performance of student groups in learning mathematics and problem solving (e.g. see [9,12,13,14,17], etc).

In this paper such kind of principles will be applied for comparing the results of performance of two groups of students of the Graduate Technological Educational Institute of Western Greece concerning their comprehension of real numbers in general and irrational numbers in particular. The first group was taught the subject in the traditional way (control group), while the APOS/ACE instructional treatment was applied for the second group (experimental group). The APOS/ACE theory for learning and teaching mathematics was developed during the 1990's in the USA by a team of mathematicians and mathematics educators led by Ed Dubinsky and one of its central ideas is the use of computers as a teaching tool (see [1,2]).

The rest of the present paper is formulated as follows: In the next (second) section the headlines of the APOS/ACE theory are presented and some simple examples are provided for its better understanding. In the third section our classroom experiment is described, while in the fourth section a simple fuzzy model is applied on our experiment's process and the centroid defuzzification technique is used for assessing the performance of the two groups. Finally, in the last (fifth) section our conclusions are stated and the future perspectives of this research are discussed.

For general facts on fuzzy sets we refer to the book [4]. 


\section{The APOS/ACE Theory for Learning/Teaching Mathematics}

APOS is a theory based on Piaget's principle that an individual learns (e.g. mathematics) by applying certain mental mechanisms to build specific mental structures and uses these structures to deal with problems connected to the corresponding situations [7]. Thus, according to the APOS analysis, an individual deals with a mathematical situation by using certain mental mechanisms to build cognitive structures that are applied to the situation. The main mechanisms are called interiorization and encapsulation and the related structures are Actions, Processes, Objects and Schemas. The last four words constitute the acronym APOS.

The theory postulates that a mathematical concept begins to be formed as one applies transformations on certain entities to obtain other entities. A transformation is first conceived as an action. For example, if an individual can think of a function only through an explicit expression and can do little more than substitute for the variable in the expression and manipulate it, he/she is considered to have an action understanding on functions.

As an individual repeats and reflects on an action it may be interiorized to a mental process. A process performs the same operation as the action, but wholly in the mind of the individual enabling her/him to imagine performing the transformation without having to execute each step explicitly. For example, an individual with a process understanding of a function thinks about it in terms of inputs, possibly unspecified, and transformations of those inputs to produce outputs.

If one becomes aware of a mental process as a totality and can construct transformations acting on this totality, then we say that the individual has encapsulated the process into a cognitive object. In case of functions encapsulation allows one to form sets of functions, to define operations on such sets, to equip them with a topology, etc. Although a process is transformed into an object by encapsulation, this is often neither easy nor immediate. This happens because encapsulation entails a radical shift in the nature of one's conceptualization, since it signifies the ability to think of the same concept as a mathematical entity to which new, higher-level transformations can be applied. On the other hand, the mental process that led to a mental object through encapsulation remains still available and many mathematical situations require one to de-encapsulate an object back to the process that led to it. This cycle may be repeated one or more times. For example, in defining the sum $\mathrm{f}+\mathrm{g}$ of two functions possessing a common domain, say $A$, it is necessary to reconsider again $f$ and $g$ at a process level and thinking of all $\mathrm{x}$ in $\mathrm{A}$ to obtain a new process associating to each $\mathrm{x}$ in $\mathrm{A}$ the sum $\mathrm{f}(\mathrm{x})+\mathrm{g}(\mathrm{x})$. Then this new process must be encapsulated, in order to obtain the function $\mathrm{f}+\mathrm{g}$ at an object level.

A mathematical topic often involves many actions, processes and objects that need to be organized into a coherent framework that enables the individual to decide which mental processes to use in dealing with a mathematical situation. Such a framework is called a schema. In the case of functions it is the schema structure that is used to see a function in a given mathematical or real-world situation.
However, one must notice that there are not any rubrics in general to assess explicitly the level of understanding (by students) of mathematics corresponding to each cognitive level (structure) of the APOS theory. This is in fact a matter depending on the instructor's experience and intuition.

The APOS theory has important consequences for education. Simply put, it says that the teaching of mathematics should consist in helping students use the mental structures they already have to develop an understanding of as much mathematics as those available structures can handle. For students to move further, teaching should help them to build new, more powerful structures for handling more and more advanced mathematics. Dubinsky and his collaborators realized that for each mental construction that comes out of an APOS analysis, one can find a computer task of writing a program or code, such that, if a student engages in that task, he (she) is fairly likely to build the mental construction that leads to learning the mathematics. In other words, performing the task is an experience that leads to one or more mental constructions. As a consequence of the above finding, the pedagogical approach based on APOS analysis, known as the ACE teaching cycle, is a repeated cycle of three components: $(\boldsymbol{A})$ activities on the computer, (C) classroom discussion and (E) exercises done outside the class. The target of the activities on the computer is to help students in building the proper mental constructions for the better understanding and learning of the corresponding mathematical topic. The students discuss later in the classroom their experiences from the computer tasks performed in the laboratory, they repeat the same tasks without the help of computer and they reach, under their instructor's guidance and help, to the proper conclusions. Finally, the purpose of the exercises, which are given by the tutor as a home work, is to check and to embed better the new mathematical knowledge (for more details see $[1,2,18]$, etc).

\section{The Classroom Experiment}

\subsection{Introduction to the Experiment}

The implementation of the ACE cycle and its effectiveness in helping students making mental constructions and learn mathematics has been reported in several research studies of the Dubinsky's team. A summary of earlier work can be found in [19]. More recently this approach was applied in studying the preservice teachers understanding of the relation between a fraction or an integer and its decimal expansion (see $[20,21])$, for understanding the derivatives [5], etc.

In developing and applying in practice the ACE design for teaching the real numbers in general and the irrational numbers in particular a classroom experiment was performed with two groups of students of the Graduate Technological Educational Institute (T. E. I.) of Western Greece in Patras being at their first term of studies. Notice that the students of both groups had more or less the same mathematical background from secondary education, since they had finished the same type of Lyceum (the upper level of secondary education in Greece). Further, the 
grades that they obtained in the Panhellenic exams for entrance in the higher education were of about the same level. Also, since they were in the first term of their studies, they had attended no previous mathematical courses at the T. E. I. of Western Greece.

The subjects of the experimental group were 90 students of the School of Technological Applications (prospective engineers) attending the course "Higher Mathematics I"1. The students of this group were taught the real numbers in the computer laboratory and in the classroom according to our ACE design that we shall present below. The subjects of the control group were 100 students of the School of Management and Economics attending a similar mathematical course (the instructor was the same person). In this group the lectures were performed in the classical way on the board followed by a number of exercises and examples and without making use of computers. The students participated in solving these exercises. The teaching procedure for real numbers involved 12 didactic hours (45 minutes each) for both groups covering exactly the same topics. The only difference was that half of these hours were spent for the students of the experimental group in the computer laboratory according to our APOS/ACE design.

On the first day in class the students of both groups completed individually a five-item pre-instructional written questionnaire. This questionnaire is presented in Appendix I at the end of the paper, where it is also mentioned what each of its five questions reflects. We believe that the combination of all these questions, which covers the whole knowledge about real numbers taught at high school, hits the target of the pre-instructional questionnaire, which was to allow the students to demonstrate if they had satisfactorily acquired or not this knowledge. The instrument served to establish the similarity of the two groups and to guide the development of the teaching process. In order to give them an extra motivation, they were advised to perform carefully the questionnaire, since their performance to it is counted towards their grade in the course. The results of the preinstructional test for the two student groups are presented in Table 1.

Table 1. Results of the pre-instructional test

\begin{tabular}{|c|c|c|c|}
\hline \multicolumn{5}{|c|}{ Experimental group $\left(\mathrm{G}_{1}\right)$} \\
\hline \% Scale & Grade & Amount of students & \% of students \\
\hline $89-100$ & A & 0 & 0 \\
\hline $77-88$ & B & 17 & 18.9 \\
\hline $65-76$ & C & 18 & 20 \\
\hline $53-64$ & D & 25 & 27.8 \\
\hline Less than 53 & F & 30 & 33.3 \\
\hline Total & \multicolumn{4}{|c|}{ Control group $\left(\mathrm{G}_{2}\right)$} \\
\hline \multicolumn{5}{|c|}{ \% } & 0 & 0 \\
\hline \% Scale & Grade & Amount of students & \% of students \\
\hline $89-10$ & A & 18 & 18 \\
\hline $77-88$ & B & 20 & 30 \\
\hline $65-76$ & C & 30 & 32 \\
\hline 53-64 & D & 32 & \\
\hline Less than 53 & F & 100 & \\
\hline Total & & &
\end{tabular}

${ }^{1}$ This course involves an introductory chapter repeating and extending the students' knowledge from secondary education about the basic sets of numbers, Complex Numbers, Differential and Integral Calculus in one variable, Elementary Differential Equations and Linear Algebra.
Our APOS/ACE approach for teaching the real numbers to the students of the experimental group involved three iterations of the ACE cycle. Each cycle consisted of two class sessions, one for computer activities and one for classroom discussions. Homework exercises were assigned and collected. Notice that, since the proper understanding of the rational numbers is an essential preassumption for the comprehension of the irrational numbers, our design involved frequent repetitions of the corresponding situations for rational numbers. Some of these repetitions were adapted from [20].

\subsection{First Iteration of the ACE Cycle}

In an action level the concept of an infinite decimal (rational or irrational number) is understood by considering its finite decimal approximations. The target of the first iteration of the ACE cycle was to facilitate the interiorization of this action to a process. In fact, a number of students considered an infinite decimal as being equal to its finite decimal approximation provided by a calculator ((e.g. the quotient of the division 5:7 as being equal to 0.71428571428 ), or that $\pi=3.14$, etc (pre-state condition). The post-state target was to clarify such kind of confusions. The students completed, in the computer laboratory, activities with a preloaded decimal expansion package. They developed general descriptions of what was stored and answered various questions about an infinite digit string such as: What is a repeating decimal? Which of the strings are repeating decimals? What are the digits in the first 20 places after the decimal point and what would appear in the $1005^{\text {th }}$ place? Further, students were asked to calculate the successive finite decimal approximations of several square roots with gradually increasing accuracy.

In the classroom discussion the students reported their group responses in the computer laboratory and the class negotiated agreements. A notational system for infinite decimals was devised. For example, since $1<\sqrt{2}<2$, $1.4<\sqrt{2}<1.5,1.41<\sqrt{2}<1.42,1.414<\sqrt{2}<1.415$, $1.4142<\sqrt{2}<1.4143,1.41421<\sqrt{2}<1.41422$, etc, $\sqrt{2}$ can be written as $\sqrt{2}=1.41421 \ldots \ldots$. The dots at the end indicate that the sequence of the decimal digits is continued to infinity. Therefore, by accepting this symbolic representation of an infinite decimal we can not see written all its decimal digits. We can only see the digits of its given decimal approximation each time. The instructor recalled at this point that a repeating decimal (rational number) can be written in the form $a, b \bar{c}$. Here $a, b, c$ are non-negative integers having one or more digits, where $a$ denotes the integer part of the rational number, $b$ is its decimal portion that possibly appears before the repeating cycle (in case of mixed periodic numbers) and $c$ is the repeating cycle (period) of the number. A finite decimal can be written as a repeating decimal with period 0 or 9 . For example, $2.5=2.5 \overline{0}=2.4 \overline{9}$. The exercises included problems where certain information about an infinite digit string was provided that was sufficient to specify the string.

\subsection{Second Iteration of the ACE Cycle}

The target of the second iteration of the ACE cycle was to facilitate the encapsulation of the concept of a real 
number to a mental object. In fact, a number of students at this stage still considered that fractions, roots and decimals are different kinds of real numbers, not connected to each other (pre-state condition). Our target was to help them to understand that all these numbers are actually the same numbers written in different alternative forms and therefore to be able to consider the set $\boldsymbol{R}$ of real numbers as a totality and to perform transformations on this totality (post-state condition).

During the computer activities students were asked to work out examples with transparent and opaque decimal representations of real numbers like the following: The rational numbers $\frac{3}{5}=0.6, \frac{1}{3}=0.33 . ., \frac{281849}{99900}=$ 2.82113113113... , have transparent decimal representations, since we can foresee their decimal digits in all places; but the same is not happening with $\frac{1}{1861}=$ $0.0005373 . .$. , which, possessing a period of 1860 digits, has an opaque decimal representation. Notice that decimal representations of certain irrational numbers, despite of their complex structure in general, are also transparent. For example, this happens with the numbers $2.001313113111311113111113 \ldots$ where 1 , following 13 , is repeated one more time at each time, and 0.282288222888222288882 ... where 2 and 8 , following 28 , are repeated one more time at each time (for more details on the transparent and the opaque representations of the real numbers see, for example, [22]).. Taking this opportunity the instructor clarified to the class that an infinite decimal is an incommensurable (non-periodic) decimal not because its decimal digits are not repeated in a concrete process (this in fact can happen according to the above two examples), but because it has no period, i.e. its decimal digits are not repeated in the same concrete series. Some standard cases of decimal expansions of transcendental numbers like $\pi$ and $e$ were also added to the above examples. Students were also asked to convert fractions and roots of second or higher order to decimals and vice versa. Further, the computer activities included arithmetic operations among irrational and rational numbers by using their finite decimal approximations.

In the classroom the students performed the same mathematical activities without using the computers. In this way they realized that in converting a fraction to a decimal, if the quotient obtained is an infinite decimal having a long period, a long and laborious division is reached in general, which is not possible to be determined soon. At this point the instructor emphasized that given a

fraction $\frac{\mu}{v}, \mu, v \in Z, v \neq 0$, the quotient of the division $\mu: v$ is always a periodic decimal. The probability to be a finite decimal is small enough, since a fraction, whose denominator is not a product of powers of 2 and/or 5 , cannot be written as a finite decimal. In case of an infinite decimal, since the remainder of the division $\mu: v$ is smaller that $v$, performing the division and after a finite number of steps (at most $v$-1) the same remainder will reappear at some step. This means that the resulting decimal is a periodic one, having a period of at most $v-1$ digits. Conversely, a standard method for converting periodic numbers to fractions (although other methods could be used as well) is by subtracting both members of proper equations containing multiples of a power of 10 of the given number. For example, given $x=2.75323232 \ldots$, we write $10000 x=27532.3232 \ldots$ and $100 x=275.3232 \ldots$, wherefrom we get that $9900 x=27532-275$, or $x=\frac{27257}{9900}$.

Reflecting on the above examples the students reached to the conclusion that periodic decimals and fractions are the same numbers written in a different way. Students' contact at school with the definition of irrational numbers as incommensurable decimals is usually rather slim, while emphasis is given on defining them as non-rational numbers (i.e. they cannot be written as fractions $\frac{\mu}{v}$, with $\mu, v$ integers and $v \neq 0$ ). However, students must clearly understand the equivalence between the above two definitions: Similarly to the fact that rational numbers and periodic decimals are the same numbers written in a different way, the same holds for non-rational numbers and incommensurable decimals. Thus, the set of real numbers $\boldsymbol{R}$ can be defined as the set of all commensurable and incommensurable decimals and their opposites, which are negative numbers (e.g. the opposite of 11.5 is -11.5 , etc). In closing the classroom discussion the instructor presented empirically the concept of a sequence of finite decimals and of its limit (i.e. what it means to "tend" to a number) and explained it to students by using the appropriate examples, like this with $\sqrt{2}$ mentioned above. In no case is it necessary for the teacher to give the analytic definition of the limit of a sequence. The above empiric approach is enough for helping students to encapsulate the concept of a real number to a mental object. The homework exercises were standard problems related to the topics mentioned above aiming to consolidate the students' knowledge and understanding of these topics.

\subsection{Third Iteration of the ACE Cycle}

The target of the third iteration of the ACE cycle was to help students to enlist real numbers in general and irrational numbers in particular in their cognitive schema related to the already known basic sets of numbers, i.e. natural numbers, integers and rational numbers (post-state condition). A prerequisite for this is that they must be able to transfer in comfort among the several representations of real numbers, which was not the case for many students (pre-state condition) before the third iteration of the ACE cycle.

The computer activities in this cycle involved among others examples of constructions of line segments with incommensurable lengths; either classical geometrical constructions by using the Pythagorean theorem, like $\sqrt{2}, \sqrt{3}, \sqrt{5}$ etc, or cases where the construction of the graph of a function is necessary, like $\sqrt[3]{2}$ with the function $f(x)=\sqrt[3]{x}$ (or $f(x)=\sqrt[3]{x}-2$ ) etc. They involved also examples of writing real numbers in the form of a series $x=\sum_{n=0}^{\infty} \frac{\kappa_{n}}{10^{n}}$, where $\kappa_{0}$ is an integer and $\kappa_{1}, \kappa_{2}, \ldots, \kappa_{n}, \ldots$. are natural numbers less than 10 . Finally, the computer activities involved also examples of interpolation of rational and irrational numbers between 
two given integers, or between two rational (irrational) numbers aiming to promote the later discussion in classroom about the density of the sets of rational and real numbers.

In the classroom discussion the instructor recalled first that in defining the set $\mathbf{Q}$ of rational numbers as the set of all fractions and in order to count each fraction only once, we considered only the fractions of the form $\pm \frac{\mu}{v}$, where $\mu$ and $v$ are non negative integers $(v \neq 0)$, with greatest common divisor equal to 1 . In an analogous way, since for all integers $\kappa$ and $\alpha$ with $1 \leq \alpha \leq 9$ we have $\kappa . \alpha=\kappa$. $(\alpha$ 1) $\overline{9}$ and $\kappa \cdot \overline{9}=\kappa+1$, in defining $\boldsymbol{R}$ as the set of all decimals and in order to count each real number only once, we must exclude all infinite decimal expansions of the form $\kappa \cdot \kappa_{1} \kappa_{2} \ldots \ldots \ldots$, in which there exists a natural number $v$ such that $\kappa_{\mu}=9$ for all $\mu \geq v$.

Another crucial matter for the instructor is to find the proper way to explain to students the continuum of $\boldsymbol{R}$ with respect to the countability of $\boldsymbol{Q}$ (the latter was taught in the course within the general repetition performed for the rational numbers). In other words to persuade them that in a given interval of numbers it is possible to have an infinite number of elements of a certain type (rational numbers) and at the same time to be able to add an infinity of elements of another type (irrational numbers), when this is not compatible with the usual logic and intuition. It seems that the use of the geometric representations of real numbers is a proper way to deal with this problem (an interval of points on the real axis cannot be "filled" with rational points only). The difficulty in this case is that most of the irrational numbers, like $\sqrt[3]{2}, \pi$, e, etc, are associated with lengths of line segments that cannot be constructed geometrically. Therefore, to all these numbers points of the real axis are corresponded in an approximate way by using their finite decimal approximations and our fantasy.

However, things became more complicated when we arrived to the natural, but crucial, question asked by students: "Which numbers can be written in the form of an incommensurable decimal number?" At the lower highschool level (Gymnasium) students learnt that this happens with the square roots of positive rational numbers that they cannot be exactly determined (i.e. they have not an exact value). Later, at the upper high-school level (Lyceum), they learnt that this also happens with the n-th roots, $n \in \mathbf{N}, n \geq 2$. However, as the instructor emphasized at this point, the converse is not true, since they are incommensurable decimal numbers that cannot be written as roots, or in a more general expression they are not roots of an algebraic equation with rational coefficients. Thus we arrive to the concept of the transcendental numbers. This new kind of numbers usually activates students' imagination and increases their interest by creating a pedagogical atmosphere of mystery and surprise. The instructor informed students (without giving any proofs) that the set of algebraic numbers is a denumerable set, while, as Cantor has proved, the set of transcendental numbers has the power of continuum (i.e. it is equivalent with the whole set $\boldsymbol{R}$ of real numbers, "filling" all the points of the real axis). This practically means that transcendental numbers are much more than algebraic numbers, but, apart from some characteristic examples, like $\pi$ and e, the information that we have about them is very small relative to their multitude.

\subsection{The Post-instructional Test}

Our general didactic approach included a fertile utilization of already existing informal knowledge and beliefs about numbers, active learning through rediscovery of concepts and conclusions, construction of knowledge by students individually or as a team in the computer laboratory and in classroom. The teaching process was based on multiple representations of real numbers and on flexible transformations among them (for more details see [18]). Notice that, due to the limited time available for teaching the real numbers within the general mathematical course of the first term of studies (involving many different to each other topics), the computer activities designed for the corresponding ACE cycle didn't involve any program or code writing, as Dubinsky and his collaborators suggest (see last paragraph of section 2); ready mathematical software was only used. However, as the results of our experiments showed (see below), this was enough to help students in building a powerful schema for real numbers.

At the end of the instructional unit the students of both groups completed a new ten-item post-instructional written questionnaire presented in Appendix II at the end of the paper. In this appendix it is also mentioned what each one of the questions reflects. The whole set of these questions covers almost all the material about real numbers taught at the course through the described in sections 3.2 to 3.4 three iterations of the ACE cycle. Thus, a student who obtained a satisfactory score in this test, has very probably achieved an understanding of real numbers at an object's level and has enlisted them successfully in his/her cognitive schema of all basic sets of numbers. Therefore, the post-instructional test is a "teller" of the competence and understanding of the real numbers by students. Students were instructed to work on the questionnaire individually and to answer each question thoroughly. The instrument counted as a progress grade added to the course's final exam results.

Table 2. Results of the post-instructional test

\begin{tabular}{|c|c|c|c|}
\hline \multicolumn{4}{|c|}{ Experimental group $\left(\mathrm{G}_{1}\right)$} \\
\hline$\%$ Scale & Grade & Amount of students & $\%$ of students \\
\hline $89-100$ & A & 3 & 3.3 \\
\hline $77-88$ & $\mathrm{~B}$ & 15 & 16.7 \\
\hline $65-76$ & $\mathrm{C}$ & 34 & 37.8 \\
\hline $53-64$ & $\mathrm{D}$ & 22 & 24.4 \\
\hline Less than 53 & $\mathrm{~F}$ & 16 & 17.8 \\
\hline Total & & 90 & \\
\hline \multicolumn{4}{|c|}{ Control group $\left(\mathrm{G}_{2}\right)$} \\
\hline$\%$ Scale & Grade & Amount of students & $\%$ of students \\
\hline $89-100$ & A & 1 & 1 \\
\hline $77-88$ & B & 10 & 10 \\
\hline $65-76$ & $\mathrm{C}$ & 37 & 37 \\
\hline $53-64$ & $\mathrm{D}$ & 31 & 31 \\
\hline Less than 53 & $\mathrm{~F}$ & 21 & 21 \\
\hline Total & & 100 & \\
\hline
\end{tabular}

There were three weeks between the pre-test and posttest (4 didactic hours per week X 3 weeks $=12$ in total didactic hours) and no additional learning supports apart from those described above provided by the instructor to 
each group during this period. The results of the postinstructional test for the two student groups are presented in Table 2.

\section{Application of Fuzzy Logic in Assessing the Student's Performance}

As already stated in our introduction, principles of fuzzy logic were applied for comparing the performance of the two groups of students of the previous experiment by implementing the following fuzzy model on the experiment's process and the defuzzification technique known as the centroid method. According to this method, the centre of gravity of the graph of the membership function involved provides an alternative measure of the system's performance. The application of the centroid method in practice is simple and evident and, in contrast to the measures of uncertainty which can be also used as alternative defuzzification techniques (for example see [12] and its references), needs no complicated calculations in its final step. The techniques that will be applied here have been also used earlier in $[8,14,15]$, etc.

Given a fuzzy subset $A=\{(x, m(x)): x \in U\}$ of the universal set $U$ of the discourse with membership function $m: U \rightarrow[0,1]$, an interval of values from a prefixed numerical distribution is associated to each $x \in U$, which actually means that $U$ is replaced with a set of real intervals. Then, the graph of the membership function $y=m(x)$ is constructed. There is a commonly used approach in fuzzy logic to measure performance with the pair of numbers $\left(\mathrm{x}_{\mathrm{c}}, \mathrm{y}_{\mathrm{c}}\right)$ as the coordinates of the centre of gravity (centoid), say $\mathrm{F}_{\mathrm{c}}$, of the level's section $F$ contained between this graph and the $0 \mathrm{X}$ axis, which can be calculated using the following well-known from Mechanics formulas:

$$
x_{C}=\frac{\iint_{F} x d x d y}{\iint_{F} d x d y}, y_{C}=\frac{\iint_{F} y d x d y}{\iint_{F} d x d y}
$$

Concerning the described experiment, a student's performance was characterized as very low (F) if $x \in[0,1)$, as low (D) if $x \in[1,2)$, as intermediate (C) if $x \in[2,3)$, as high (B) if $x \in[3,4)$ and as very high (A) if $x \in[4,5]$ respectively. Denote by $G_{1}$ the experimental group and by $\mathrm{G}_{2}$ the control group and set $U=\{\mathrm{A}, \mathrm{B}, \mathrm{C}, \mathrm{D}, \mathrm{F}\}$.

We are going to represent the $G_{i}$ 's, $i=1,2$, as fuzzy subsets of $U$. For this, if $n_{i F}, n_{i D} n_{i C}, n_{i B}$ and $n_{i A}$ denote the number of students of group $G_{i}$ who achieved very low, low, intermediate, high and very high success respectively, the membership function $\mathrm{m}_{\mathrm{Gi}}$ is defined in terms of the frequencies, i.e. by

$$
\mathrm{m}_{\mathrm{Gi}}(\mathrm{x})=\frac{n_{i x}}{n}
$$

for each $\mathrm{x}$ in $U$. Thus one can write

$$
\mathrm{G}_{\mathrm{i}}=\left\{\left(\mathrm{x}, \frac{n_{i x}}{n}\right): \mathrm{x} \in U\right\}, \mathrm{i}=1,2 .
$$

Therefore in this case the level's section $F$ defined by the corresponding fuzzy subset of $U$ is the bar graph of Figure 1 consisting of five rectangles, say $F_{i}, \mathrm{i}=1,2,3,4,5$, whose sides lying on the $\mathrm{x}$ axis have length 1 . In this case $\iint_{F} d x d y$ is the area of $F$ which is equal to $\sum_{i=1}^{5} y_{i}$. Also $\iint_{F} x d x d y=\sum_{i=1}^{5} \iint_{F_{i}} x d x d y=\sum_{i=1}^{5} \int_{0}^{y_{i}} d y \int_{i-1}^{i} x d x=\sum_{i=1}^{5} y_{i} \int_{i-1}^{i} x d x=$ $\frac{1}{2} \sum_{i=1}^{5}(2 i-1) y_{i} \quad, \quad$ and $\quad \iint_{F} y d x d y=\sum_{i=1}^{5} \iint_{F_{i}} y d x d y$ $=\sum_{i=1}^{5} \int_{0}^{y_{i}} y d y \int_{i-1}^{i} d x=\sum_{i=1}^{n} \int_{0}^{y_{i}} y d y=\frac{1}{2} \sum_{i=1}^{n} y_{i}^{2}$.

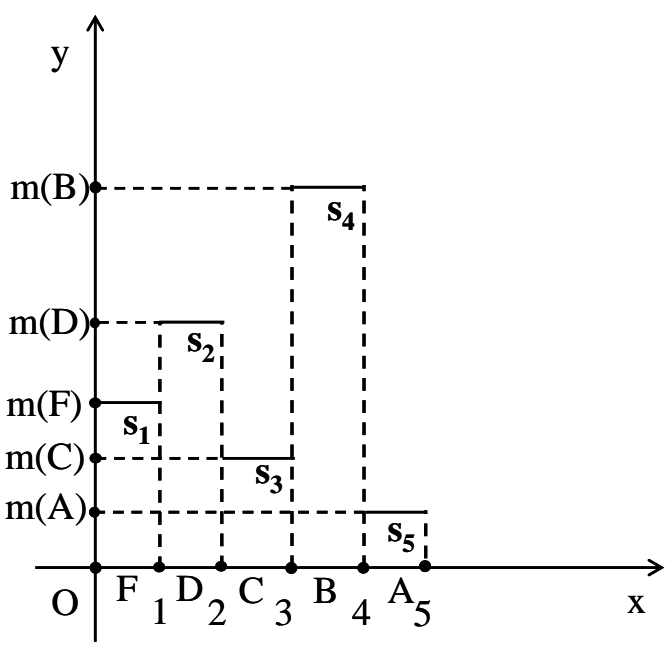

Figure 1. Bar graphical data representation

Therefore formulas (1) are transformed into the following form:

$$
\begin{aligned}
& x_{C}=\frac{1}{2}\left(\frac{y_{1}+3 y_{2}+5 y_{3}+7 y_{4}+9 y_{5}}{y_{1}+y_{2}+y_{3}+y_{4}+y_{5}}\right), \\
& y_{C}=\frac{1}{2}\left(\frac{y_{1}^{2}+y_{2}^{2}+y_{3}^{2}+y_{4}^{2}+y_{5}^{2}}{y_{1}+y_{2}+y_{3}+y_{4}+y_{5}}\right) .
\end{aligned}
$$

Normalizing our fuzzy data by dividing each $\mathrm{m}(\mathrm{x})$, $\mathrm{x} \in U$, with the sum of all membership degrees we can assume without loss of the generality that $y_{1}+y_{2}+y_{3}+y_{4}+y_{5}$ $=1$. Therefore one can write:

$$
\begin{aligned}
& x_{C}=\frac{1}{2}\left(y_{1}+3 y_{2}+5 y_{3}+7 y_{4}+9 y_{5}\right), \\
& y_{c}=\frac{1}{2}\left(y_{1}^{2}+y_{2}^{2}+y_{3}^{2}+y_{4}^{2}+y_{5}^{2}\right) .
\end{aligned}
$$

with $y_{i}=\frac{m\left(x_{i}\right)}{\sum_{x \in U} m(x)}$.

But $0 \leq\left(\mathrm{y}_{1}-\mathrm{y}_{2}\right)^{2}=\mathrm{y}_{1}{ }^{2}+\mathrm{y}_{2}{ }^{2}-2 \mathrm{y}_{1} \mathrm{y}_{2}$, therefore $\mathrm{y}_{1}{ }^{2}+\mathrm{y}_{2}{ }^{2}$ $\geq 2 \mathrm{y}_{1} \mathrm{y}_{2}$, with the equality holding if, and only if, $\mathrm{y}_{1}=\mathrm{y}_{2}$. In the same way one finds that $\mathrm{y}_{1}{ }^{2}+\mathrm{y}_{3}{ }^{2} \geq 2 \mathrm{y}_{1} \mathrm{y}_{3}$, and so on. Hence it is easy to check that $\left(\mathrm{y}_{1}+\mathrm{y}_{2}+\mathrm{y}_{3}+\mathrm{y}_{4}+\mathrm{y}_{5}\right)^{2} \leq$ $5\left(\mathrm{y}_{1}{ }^{2}+\mathrm{y}_{2}{ }^{2}+\mathrm{y}_{3}{ }^{2}+\mathrm{y}_{4}{ }^{2}+\mathrm{y}_{5}{ }^{2}\right)$, with the equality holding if, and only if $\mathrm{y}_{1}=\mathrm{y}_{2}=\mathrm{y}_{3}=\mathrm{y}_{4}=\mathrm{y}_{5}$. But $\mathrm{y}_{1}+\mathrm{y}_{2}+\mathrm{y}_{3}+\mathrm{y}_{4}+\mathrm{y}_{5}=1$, therefore $1 \leq 5\left(\mathrm{y}_{1}{ }^{2}+\mathrm{y}_{2}{ }^{2}+\mathrm{y}_{3}{ }^{2}+\mathrm{y}_{4}{ }^{2}+\mathrm{y}_{5}{ }^{2}\right)$ (4), with the equality holding if and only if $\mathrm{y}_{1}=\mathrm{y}_{2}=\mathrm{y}_{3}=\mathrm{y}_{4}=\mathrm{y}_{5}=\frac{1}{5}$. 
Then the first of formulas (3) gives that $x_{c}=\frac{5}{2}$. Further, combining the inequality (4) with the second of formulas (3), one finds that $1 \leq 10 y_{c}$, or $y_{c} \geq \frac{1}{10}$. Therefore the unique minimum for $y_{c}$ corresponds to the centre of gravity $F_{m}\left(\frac{5}{2}, \frac{1}{10}\right)$.

The ideal case is when $y_{1}=y_{2}=y_{3}=y_{4}=0$ and $y_{5}=1$. Then from formulas (3) we get that $x_{c}=\frac{9}{2}$ and $y_{c}=$ $\frac{1}{2}$.Therefore the centre of gravity in this case is the point $F_{i}\left(\frac{9}{2}, \frac{1}{2}\right)$.

On the other hand, in the worst case $y_{1}=1$ and $y_{2}=y_{3}=y_{4}=y_{5}=0$. Then by formulas (3), we find that the centre of gravity is the point $F_{w}\left(\frac{1}{2}, \frac{1}{2}\right)$.

Therefore the "area" where the centre of gravity $F_{c}$ lies is represented by the triangle $F_{w} F_{m} F_{i}$ of Figure 2 .

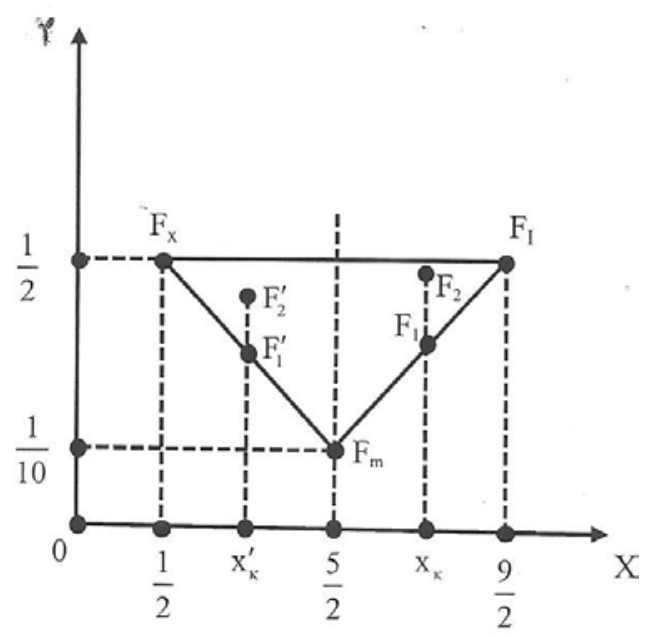

Figure 2. Graphical representation of the "area" where the centre of gravity lies

Then from elementary geometric considerations it follows that the greater is the value of $x_{c}$ the better is the group's performance. Also, for two groups with the same $x_{c} \geq 2.5$, the group having the centre of gravity which is situated closer to $F_{i}$ is the group with the higher $y_{c}$; and for two groups with the same $x_{c}<2.5$ the group having the centre of gravity which is situated farther to $F_{w}$ is the group with the lower $y_{c}$. Based on the above considerations it is logical to formulate our criterion for comparing the groups' performances in the following form:

- Among two or more groups the group with the biggest $x_{c}$ performs better.

- If two or more groups have the same $x_{c} \geq 2.5$, then the group with the higher $y_{c}$ performs better.

- If two or more groups have the same $x_{c}<2.5$, then the group with the lower $y_{c}$ performs better.

We apply now this model to the data of Table 1 (preinstructional test). The two student groups can be represented as fizzy subsets of $U$ by
$\mathrm{G}_{1}=\{(\mathrm{A}, 0),(\mathrm{B}, 0.189),(\mathrm{C}, 0.2),(\mathrm{D}, 0.278)(\mathrm{F}, 0.333)\}$

$\mathrm{G}_{2}=\{(\mathrm{A}, 0),(\mathrm{B}, 0.18),(\mathrm{C}, 0.2),(\mathrm{D}, 0.3)(\mathrm{F}, 0.32)\}$.

Then, by applying the first of formulas (3), we find that $x_{c}=0.5 *(0.333+3 * 0.278+5 * 0.2+7 * 0.189)=1.745$ for the experimental group and

$x_{c}=0.5 *(0.32+3 * 0.3+5 * 0.2+7 * 0.18)=1.74$ for the control group. So, according to the above stated criterion, the students of the two groups responded similarly to the questionnaire's items.

In the same way, on applying the model to the data of Table 2 (post-instructional test) one find that

$x_{c}=0.5 *(0.178+3 * 0.244+5 * 0.378+7 * 0.167+9 * 0.033)=$ 2.133 for the experimental group and $x_{c}=$ $0.5 *(0.21+3 * 0.31+5 * 0.37+7 * 0.1+9 * 0.01)=1.89$ for the control group. So, according to the above stated criterion, the experimental group demonstrated a significantly better performance than the control group.

An analogous experiment was also performed during the next semester with similar results. Notice also that two analogous experiments were performed during the previous academic year In this case the students' performance was assessed by applying traditional methods based on the principles of the bivalent logic and the results obtained were also similar [17].

In concluding, it seems that the application of the APOS/ACE teaching style enhances significantly the students' understanding of the real numbers in general and of the irrational numbers in particular.

REMARK: The statistical tests (average, median, standard deviation, etc), which are usually adopted as assessment methods by other authors, are mainly focusing on the students' mean performance. On the contrary, as it can be easily seen on observing the formulas (3) obtained in this section, according to the centroid defuzzification technique the higher is a student's performance the more its "contribution" to the corresponding group's total performance (weighted performance). In other words, our fuzzy method is mainly focusing on the students' quality performance. As a result, when the above different assessment methods are simultaneously applied for comparing the performance of two or more student groups the results obtained may differ to each other in certain cases. The following simplified example illustrates this possibility:

EXAMPLE: Consider the data of Table 2 and assume, with exception only of the category of grade $F$, that the students of the experimental group $\left(G_{1}\right)$ obtained the lowest possible scores in their grade categories, while the students of the control group $\left(\mathrm{G}_{2}\right)$ obtained the highest ones. Further, assume that in the grade category $\mathrm{F}$ all students of $G_{1}$ scored 30 marks, while all students of $G_{2}$ scored 50 marks. In the above hypothetical case the average score for the students of $G_{1}$ is $\frac{3 * 89+15 * 77+34 * 65+22 * 53+16 * 30}{90} \approx 58.64$, while for the students of $\mathrm{G}_{2}$ is
$\frac{1 * 100+10 * 88+37 * 76+31 * 64+21 * 50}{100} \approx 77.26$. In other words, if the average was considered as the assessment criterion of the two groups' performance, then the control group would demonstrate a much higher performance than the experimental group, which contradicts the result obtained above by the centroid method. 


\section{Discussion and conclusions}

The novelty/emphasis of this paper lies on the following two facts:

1. On the application of the APOS/ACE instructional treatment for teaching the real numbers.

2. On the use of Fuzzy Logic in assessing the students' performance.

More explicitly, the results of our classroom experiments, performed recently at the graduate T. E. I of Western Greece showed that the application of the APOS/ACE approach for teaching the real numbers in general and the irrational numbers in particular improved significantly the students' performance helping them in building effectively a powerful cognitive schema for the basic sets of numbers (including real numbers). However, all these discussed in this article about the advantages of the APOS/ACE teaching approach must not be considered as an intention to impose a model of teaching real numbers at an elementary level. On the contrary, our general belief is that the instructor should be able to make a small "local research", readapting methods and plans of the teaching process according to the teaching environment and the special conditions of each class [9]. Therefore the introduction of the APOS/ACE instructional treatment must be considered only as an effort to introduce an alternative approach that could help the instructor to reconsider and organize better his/her plans for teaching real numbers.

On the other hand, Fuzzy Logic, due to its nature of characterizing a situation with multiple values, offers a wider and richer field of resources, than the traditional assessment methods, based on principles of bivalent logic and probability, do. However, some disadvantages may also appear in adopting fuzzy techniques. Such disadvantages could be the utilization of linguistic expressions, which are usually involve a degree of uncertainty; the (non-unique) choice of the corresponding membership function, which, although it should be based on proper statistical and/or logical data, it also depends in a degree on the user's personal criteria, etc. Therefore a fuzzy model must be always checked carefully before its practical application.

The centroid defuzzification technique, which was applied in this paper as an assessment method of the students' performance, is connected to their quality (weighted) performance, in contrast to the traditional methods (statistical tests, etc), which are mainly focusing on the students' mean performance. Consequently, contradicting to each other results could appear in evaluating the students' performance by using these different approaches and this is illustrated by our example provided at the end of section 4 Therefore a simultaneous use of these approaches could help the researcher to choose the proper students' assessment method according to his/her personal priorities and criteria of goals. In concluding, the added value of the Fuzzy Logic utilization in our classroom experiment is that it provides an alternative general assessment method, which treats differently the students' performance (quality performance) with respect to what the traditional methods usually do (mean performance). Moreover, the above method (centroid technique), once developed, it is very simple to its application in practice needing no complicated calculations.

Among our future research plans is the performance of more classroom experiments with different groups of students (high school students as well!) in order to obtain statistically stronger results and conclusions. Furthermore, since the centroid deffuzzification technique is part of a general fuzzy model introduced in earlier papers [13,14,15] in dealing with situations in a system's operation characterized by a degree of fuzziness and/or uncertainty, another direction of our future research concerns the effort of representing in terms of this model even more such situations related to several sectors of the human activity (e.g. education, management, artificial intelligence, everyday life, etc).

\section{References}

[1] Asiala, M., et al. (1996), A framework for research and curriculum development in undergraduate mathematics education, Research in Collegiate Mathematics Education II, CBMS Issues in Mathematics Education, 6, 1-32.

[2] Dubinsky, E. \& McDonald, M. A. (2001), APOS: A constructivist theory of learning in undergraduate mathematics education research. In: D. Holton et al. (Eds), The Teaching and learning of Mathematics at University Level: An ICMI Study, 273-280, Kluwer Academic Publishers, Dordrecht, Netherlands.

[3] Espin, E. A. \& Oliveras, C. M. L. (1997), Introduction to the Use of Fuzzy Logic in the Assessment of Mathematics Teachers' Professional Knowledge, Proceedings of the First Mediterranean Conference on Mathematics, Cyprus, pp. 107-113.

[4] Klir, G. J. \& Folger, T. A. (1988), Fuzzy Sets, Uncertainty and Information, Prentice-Hall, London

[5] Maharaj, A. (2013), An APOS analysis of natural science students' understanding of derivatives, South African Journal of Education, 33(1), 19 pages.

[6] Perdikaris, S. (2002), Measuring the student group capacity for obtaining geometric information in the van Hiele development thought process: A fuzzy approach, Fuzzy Sets and Mathematics, 16 (3), 81-86.

[7] Piaget, J. (1970), Genetic Epistemology, Columbia University Press, New York and London.

[8] Subbotin, I. Ya. Badkoobehi, H., Bilotckii, N. N. (2004), Application of fuzzy logic to learning assessment. Didactics of Mathematics: Problems and Investigations, 22, 38-41.

[9] Voskoglou, M. Gr. (1999), The process of learning mathematics: A fuzzy set approach, Heuristic and Didactics of Exact Sciences (Ukraine), 10, 9-13,

[10] Voskoglou, M. Gr. (2009), The mathematics teacher in the modern society, Quaderni di Ricerca in Didattica (Scienze Mathematiche), University of Palermo, 19, 24-30.

[11] Voskoglou, M. Gr. (2009), Fuzzy Sets in Case-Based Reasoning, Fuzzy Systems and Knowledge Discovery, Vol. 6, 252-256, IEEE Computer Society.

[12] Voskoglou, M. Gr. (2009.), Stochastic and fuzzy models in Mathematics Education, Artificial Intelligence and Management, Lambert Academic Publishing, Saarbrucken, Germany, 2011; for more details look at http://amzn.com./3846528218 )

[13] Voskoglou, M. Gr. (2011), Fuzzy Logic and Uncertainty in Mathematics Education, International Journal of Applications of Fuzzy Sets and Artificial Intelligence, 1, 45-64.

[14] Voskoglou, M. Gr. \& Subbotin, I. Ya. (2012), Fuzzy Models for Analogical Reasoning, International Journal of Applications of Fuzzy Sets and Artificial Intelligence, 2, 19-38

[15] Voskoglou, M. Gr. (2012), A study on fuzzy systems, American Journal of Computational and Applied Mathematics, 2(5), 232240.

[16] Voskoglou, M. Gr. (2012) A fuzzy model for human reasoning, International Journal of Mathematics and Engineering with Computers, 3(2), 61-71.

[17] Voskoglou, M. Gr. (2013), Problem solving, fuzzy logic and computational thinking, Egyptian Computer Science Journal, 37(1), 131-145. 
[18] Voskoglou, M. Gr. (2013), An application of the APOS/ACE approach in teaching the irrational numbers, Journal of Mathematical Sciences and Mathematics Education, 8(1), 30-47

[19] Weller, K. et al. (2003), Students performance and attitudes in courses based on APOS theory and the ACE teaching cycle. In: A. Selden et al. (Eds.), Research in collegiate mathematics education $V$ (pp. 97-181), Providence, RI: American Mathematical Society.

[20] Weller, K., Arnon, I \& Dubinski, E. (2009), Pre-service Teachers' Understanding of the Relation between a Fraction or Integer and its Decimal Expansion, Canadian Journal of Science, Mathematics and Technology Education, 9(1), 5-28.

[21] Weller, K., Arnon, I \& Dubinski, E. (2011), Preservice Teachers' Understanding of the Relation Between a Fraction or Integer and Its Decimal Expansion: Strength and Stability of Belief, Canadian Journal of Science, Mathematics and Technology Education, 11(2), 129-159.

[22] Zadeh, L. A., Fuzzy Sets, Information and Control, 8, 338-353, 1965.

[23] Zazkis, R. \& Sirotic, N. (2010), Representing and Defining Irrational Numbers: Exposing the Missing Link, CBMS Issues in Mathematics Education, 16, 1-27.

\section{Appendix I: Pre-instructional questionnaire}

1. Which of the following numbers are natural, integers, rational, irrational and real numbers?

$$
\begin{aligned}
& -2,-\frac{5}{3}, 0,9.08,5,7.333 \ldots, \pi=3.14159 \ldots \\
& \sqrt{3}, \quad-\sqrt{4}, \quad \frac{22}{11}, \quad 5 \sqrt{3}, \quad-\frac{\sqrt{5}}{\sqrt{20}}, \\
& (\sqrt{3}+2)(\sqrt{3}-2),-\frac{\sqrt{5}}{2}, \sqrt{7}-2, \sqrt{\left(\frac{5}{3}\right)^{2}}
\end{aligned}
$$

(Checking the ability to distinguish among the different kinds of numbers)

2. Are the following inequalities correct, or wrong? Justify your answers.

$$
\frac{2}{3}<\frac{14}{21}, \frac{2002}{1001}>2
$$

(Checking the comprehension of the order in $\mathbf{Q}$ ).

3. Convert the fraction $\frac{7}{3}$ to a decimal number. What kind of decimal number is this and why we call it so? (Checking the ability of converting a fraction to a decimal and of recognizing a periodic decimal of the simplest form).

4. Between which integers and decimals with one decimal digit lies $\sqrt{2}$ ? Justify your answers (Checking the ability of the approximate calculation of a square root in terms of the corresponding definition).

5. Find two rational and two irrational numbers between 10 and 20. How many irrational numbers are there between these two integers? (Checking the comprehension of the densities of $\boldsymbol{Q}$ and $\boldsymbol{R}$ )

NOTE: In assessing the students' performance 20 marks were awarded for each completely correct answer to the above questions. However, since all questions involve two or more answers, the 20 marks were divided equivalently for each answer. For example, in question 1, involving 5 different answers, 4 marks were awarded for each correct answer. The following example illustrates the above process and helps the reader to know more about the standard of the students' responses:

EXAMPLE: The student Yannis (from the control group) answered question 1 as follows: 0 and 5 and $\frac{22}{11}=2$ are natural numbers ( 4 marks), $-2,0,5, \frac{22}{11}$ and $-\sqrt{4}=-2$ are integers (3.3 marks, since one integer was omitted), $-\frac{5}{3}, 9.08,7.333 \ldots, \frac{22}{11}$, and $\sqrt{\left(\frac{5}{3}\right)^{2}}=\frac{5}{3}$ are rational numbers (1.84 marks, since six rational numbers were omitted), $\pi, \quad \sqrt{3} \quad, \quad 5 \quad \sqrt{3}$, $-\frac{\sqrt{5}}{\sqrt{20}},(\sqrt{3}+2)(\sqrt{3}-2), \frac{\sqrt{5}}{2}, \sqrt{7}-2$ are irrational numbers (2.86 marks, since two of the above seven in total numbers are not irrational numbers) and all the given numbers are rational (4 marks). Thus Yannis obtained 16 in total marks from question 1.

\section{Appendix II: Post-instructional questionnaire}

1. Which is the exact quotient of the division $5: 7$ ? (Expected answer: The fraction $\frac{5}{7}$ or the corresponding periodic decimal, possessing a period of six digits)

2. Are 2.8254131131131... and 2.00131311311131111... periodic decimal numbers? In positive case, find the period and convert the corresponding number to a fraction (Checking the ability of distinguishing between periodic and non-periodic decimal numbers and of converting a periodic decimal to a fraction. See also the below example).

3. Find the square roots of 9,100 and 169 and describe your method of calculation (Checking the ability of calculating square roots in terms of the corresponding definition).

4. Characterize the following expressions by $\mathrm{C}$ if they are correct and by $\mathrm{W}$ if they are wrong: $\sqrt{2}=1.4$, $\sqrt{2}=1.414444 \ldots, \sqrt{2} \approx 1.41$, there is no exact value for $\sqrt{2}$ (Checking the correct writing of an irrational number) .

5. Find two rational and two irrational numbers between $\sqrt{10}$ and $\sqrt{20}$. How many rational numbers are there between these two square roots? (Checking the comprehension of the densities of $\boldsymbol{Q}$ and $\boldsymbol{R}$ ).

6. Are there any rational numbers between $\frac{1}{11}$ and $\frac{1}{10}$ ? In positive case, write down one of them. How many rational numbers are between the above two fractions? ( The same as in question 6).

7. Are there any rational numbers between 10.21 and 10.22? In positive case, write down one of them. How many rational numbers are in total between the above two decimals? ( The same as in question 6).

8. Characterize the following expressions as correct or wrong. In case of wrong ones write the corresponding correct answer. 


$$
\sqrt{3+5}=\sqrt{3}+\sqrt{5}, \sqrt{3 \cdot 7}=\sqrt{3} \cdot \sqrt{7}, \sqrt{\frac{2}{9}}=\frac{\sqrt{2}}{3}, \text { the }
$$

unique solution of the equation $x^{2}=3$ is $x=\sqrt{3}$, $\sqrt{(1-\sqrt{17})^{2}}=1-\sqrt{17}$ (Checking the ability of using correctly the properties of roots).

9. Construct, by making use of ruler and compass only, the line segments of length $\sqrt{5}$ and find the points of the real axis corresponding to the real numbers $\sqrt{5}$ and $-\sqrt{5}$. Consider a length of your choice as the unit of lengths (Checking the ability of constructing geometrically incommensurable lengths and of putting them properly on the real axis).

10. Is it possible for the sum of two irrational numbers to be a rational number? In positive case give an example
(Checking the comprehension of the fact that addition is not a closed operation in the set of irrational numbers).

NOTE: In this case 10 marks were awarded for each correct (in total) answer to the above questions (see also the note in Appendix I).

EXAMPLE: The student Maria (from the experimental group) answered the question 2 as follows: The first number is a periodic decimal with period 131, while 8254 is its non periodic decimal part (3.33 marks, since three answers are requested in total for this question). I think that the second number is a non periodic decimal, because it has no period (2 marks, because there is no proper justification of the fact that this number has no period. In fact, it could have a very long period, if its form didn't exclude this case: 13, 131, 1311, 13111, etc). Further, Maria failed to convert the first number to a fraction. Thus, she obtained 5.33 in total marks from question 2. 